\title{
Fachliches Controlling ist Führungsaufgabe
}

\section{Thomas Kaczmarek}

\begin{abstract}
Durch eine kluge Koppelung von Controlling und Qualitätsmanagement kann ein wirksames Steuerungsinstrument entstehen, welches helfen kann, das Unternehmen langfristig am Markt zu sichern. Dazu gehört, die zentralen Kernprozesse der Organisation zu identifizieren und für die daraus entstehenden Unternehmensrisiken geeignete Kennzahlen zu entwicklen. Ein solches fachliches Controlling zu konzipieren und einzuführen ist Managementaufgabe.
\end{abstract}

Die in sozialwirtschaftlichen Unternehmen wahrzunehmenden Managementaufgaben haben sich in den vergangenen Jahren verändert und erweitert, wie besonders eindrucksvoll in pflegerischen Diensten und Einrichtungen gezeigt werden kann.

Ausgelöst wurden diese Veränderungen zum einen durch den zunehmenden und politisch gewollten Wettbewerb, der in der Pflege erheblich ausgeprägter ist, als in nahezu allen anderen sozialen Dienstleistungsbereichen. Zum anderen haben auch die gesellschaftliche Diskussion um den so genannten Pflegenotstand und die mit dieser Diskussion einher gehende wachsende Reglementierung des Pflegeprozesses selbst die Anforderung an das Management von Unternehmen verändert, die in der Pflege tätig sind - die Steuerung des Kernprozesses Pflege hat an Bedeutung gewonnen.

Die Implementierung von Controlling und von Qualitätsmanagement sind zwei zentrale Antworten, mit denen den Herauforderungen durch Wettbewerb, Kostensenkungsdruck und Prüfungsintensität begegnet werden soll. Nahezu alle Pflegeunternehmen wenden deshalb Instrumente des Controllings an und implementieren ein Qualitätsmanagement.

Trotzdem gelingt es in vielen Fällen nicht, Schadensersatzansprüche und Imageschäden durch »schlechte« Pflege zu vermeiden, im Gegenteil führen Probleme bei der Dienstleistungserbringung zu finanziellen Schwierigkeiten etwa durch unverhältnismäßig hohe Personalkosten oder eine zu geringe Auslastung.

Eine Ursache dieser Schwierigkeiten kann die Entkoppelung von Controlling und Qualitätsmanagement sein. So beschränkt sich das Controlling häufig auf die Analyse der finanzwirtschaftlichen Ergebnisse der unternehmerischen Tätigkeit, im Mittelpunkt der Betrachtung stehen die Kostenstrukturen und die Ertragslage des Unternehmens.

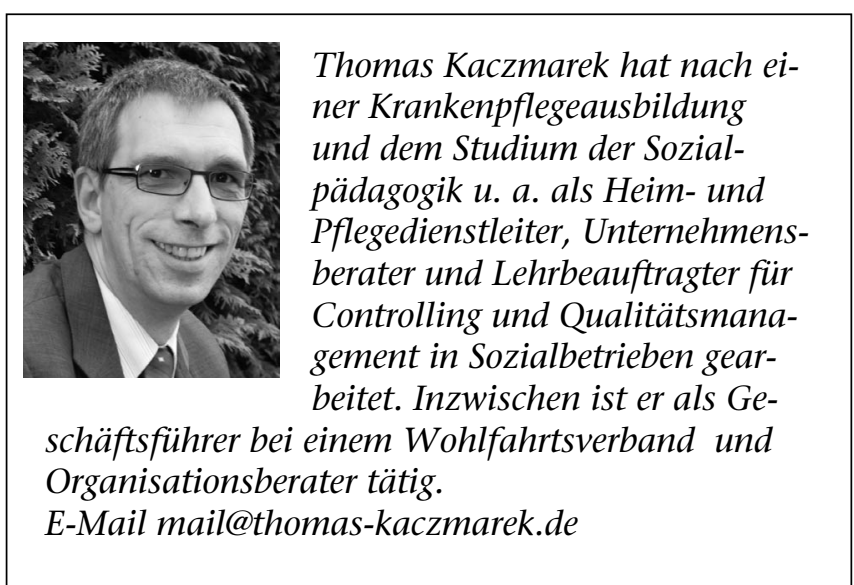

Das Qualitätsmanagement dagegen regelt unterschiedlichste Prozesse und befasst sich nicht zuletzt mit der Erfüllung verschiedenster systemimmanenter Vorgaben. Dies sind die Prüfkriterien vom Medizinischen Dienst der Krankenkassen (MDK) und von der Heimaufsicht, trägerinterne Vorlieben, dazu möglicherweise Zertifizierungsvorgaben oder verbandsübergreifend zu implementierende Systemvorgaben.

Qualitätsmanagementsysteme wirken sich jedoch häufig nicht wesentlich auf die Qualität aus, inzwischen lässt sich belegen, dass die Alltagserfahrung von Pflegekräften richtig ist: Qualitätsmanagementsysteme in der Pflege legen oft eine Vielzahl von Verfahren fest, die dann jedoch nicht in operatives Handeln übertragen werden. Es entsteht eine ressourcenvernichtende Pseudo-Realität mit hohem Frustrationspotenzial (Saßen, Sascha 2008: Qualitätsmanagement. Umsetzung in der Altenpflege. Tectum-Verlag. Marburg). Insofern liefern Qualitätsmanagementsysteme dem Management auch nur selten steuerungsrelevante inhaltliche Informationen, die sich beispielsweise auf die haftungsrelevanten Risiken der Dienstleistungserbringung beziehen.

Im Ergebnis schützt auch ein zertifiziertes und MDK-geprüftes Qualitätsmanagementsystem nicht automatisch vor existenzbedrohenden fachlichen Fehlleistungen. Die Beschaffung der zur fachlichen Steuerung und Risikoabwägung erforderlichen Informationen muss dem Controlling obliegen: Die Aufgabe des Controllings besteht darin, die Unternehmensführung bei der Wahrnehmung ihrer planenden, steuernden, koordinierenden und kontrollierenden Aufgaben zu beraten und zu unterstützen (Peters, 
Andre/Moos, Gabriele (2008): BWL für soziale Berufe. Ernst Reinhardt Verlag. München).

Will das Controlling die steuerungsrelevanten fachlichen Informationen haben, so muss es als ein fachliches Controlling die Wirkung der Qualitätsmanagementmaßnahmen in bestimmten Bereichen erfassen und abbilden. Ein so verstandenes fachliches Controlling verbindet das finanzwirtschaftliche Controlling mit dem Qualitäts- und dem Risikomanagement und liefert der Leitung Entscheidungsgrundlagen, insofern ist die Implementierung eines fachlichen Controllings auch eine Managementaufgabe.

Dabei sollen die Instrumente des Qualitätsmanagement zur Systemprüfung (z. B. Audits) nicht ersetzt oder unter einem anderen Namen wiederholt werden, ausreichend ist vielmehr die kennzahlenbasierte Abbildung einiger weniger Kernprozesse. Erreicht werden kann somit, dass der Unternehmensführung die steuerungsrelevanten Informationen verfügbar sind, die sich auf zentrale Risiken und Koordinationsbedarfe beziehen.

\section{Kernprozesse mit den größten Risiken identifizieren}

Erforderlich ist die Konzentration der Aktivitäten des fachlichen Controllings auf diejenigen Kernprozesse, welche für das Unternehmen die größten Risiken bedeuten und am häufigsten Gegenstand externer Prüfungen sind. Bei der Pflege handelt sich dabei um die Prozesse Sturzprävention, Dekubitusprävention, gewaltfreie Pflege, Prävention von Fehl- und Mangelernährung und professionelle Fallarbeit (Saßen, Sascha; Borutta, Manfred; Lennefer, Joachim (2007): Risikomanagement. Führungsstrategien für pflegerische Kernbereiche. Vincentz Verlag. Hannover).
Die Betrachtung dieser fünf Prozesse ermöglicht unmittelbare Rückschlüsse sowohl auf die Ergebnisqualität, als auch auf eventuell mit der Dienstleistung verbundene (Haftungs-) Risiken. Zwar werden im Rahmen der Qualitätsmanagementvorgaben an verschiedenen Stellen Regelungen zu den genannten Prozessen getroffen, doch kann deren zusammenfassende Betrachtung innerhalb des Qualitätsmanagements nur in Zusammenhang mit MDK-Prüfungen oder Audits erfolgen und ist insofern sehr aufwendig. Das Management braucht aber ständig kontinuierliche und zeitnahe Informationen zu den Prozessen im Rahmen des Controllings, vergleichbar mit der regelmäßigen finanzwirtschaftlichen Betrachtung durch betriebswirtschaftliche Auswertungen.

\section{Gebraucht werden Kennzahlen, die "smart« sind}

Bei der Beschaffung dieser Informationen ist unbedingt zu beachten, dass keine Parallelstruktur zum Qualitätsmanagement aufgebaut wird, zudem muss die Betrachtung

\section{Die fünf Kernprozesse in der Pflege}
1. Sturzprävention
2. Dekubitusprävention
3. gewaltfreie Pflege
4. Prävention von Fehl- und Mangelernährung
5. professionelle Fallarbeit

\begin{tabular}{|c|c|c|}
\hline Grundanliegen & Qualitătsanforderungen & Prufkriterien \\
\hline $\begin{array}{l}\text { 3. Ziel- und wirkungsorientierung } \\
\text { Wirkungsorientierung stellt beabsichtigte } \\
\text { und erzielte Effekte der Sozialen Arbeit in } \\
\text { den Vordergrund. Sie zwingt dazu, klare } \\
\text { Ziele zu definieren und Erfolgs-/ Ergebnis- } \\
\text { kriterien zu beschreiben. } \\
\text { Erklärung/Begründung } \\
\text { Wirkungsorientierung ist ein systemorien- } \\
\text { tierter Entwicklungsansatz, die Gestaltung } \\
\text { des Leistungsgeschehens gemeinsam vorzu- } \\
\text { nehmen - sowohl bezogen aut das Indivi- } \\
\text { duum, als auch aut das ihn umgebende } \\
\text { System bis hin zum Gemeinwesen. }\end{array}$ & $\begin{array}{l}\text { - Die Organisationsziele werden mit Blick auf die Wir- } \\
\text { kungsziele formuliert. } \\
\text { - Zur Erreichung der Wirkungsziele erfolgt eine insti- } \\
\text { tutionalisierte Vernetzung. } \\
\text { - Das Handeln der Dienstleistungsorganisation wird } \\
\text { zielgerichtet geplant und umgesetzt. } \\
\text { - Die formulierten Ziele müssen messbar sein. } \\
\text { - Die Erreichung der Wirkungs- und Organisationszie- } \\
\text { le wird systematisch überprütt/evaluiert. } \\
\text { - Die Ergebnisse der Evaluationen sind Bestandteil des } \\
\text { Qualitätscontrollings' und der Planung des weiteren } \\
\text { Handelns. }\end{array}$ & $\begin{array}{l}\text { - Der Regelkreislauf aus Zielformulierung, Maßnahmenpla- } \\
\text { nung, Umsetzung, Evaluation/Messung der Ergebnisse und } \\
\text { erneuter Zieltormulierung ist als systematisches Verfahren } \\
\text { dokumentiert und in der Praxis nachweisbar. } \\
\text { - Messgrößen sind definiert. } \\
\text { - Die dabei gewonnenen Daten fließen in das ständige Quali- } \\
\text { tätscontrolling sowie in das jährliche Managementreview ein } \\
\text { und unterliegen einer Aus- und Bewertung. } \\
\text { - Verbesserungsmaßnahmen aus der Bewertung der Ergebnis- } \\
\text { qualität sind sowohl im individuellen Dienstleistungsprozess } \\
\text { nachweisbar als auch auf Ebene der Organisation. } \\
\text { - Die Organisation kann Aussagen machen über erzielte Ef- } \\
\text { tekte auf der Ebene des individuellen Hilfeprozesses, der } \\
\text { Organisation sowie des Gemeinwesens. }\end{array}$ \\
\hline
\end{tabular}

Die Orientierung an den Bedürfnissen und Wünschen der Nutzer, an den vereinbarten Zielen und den erwünschten Wirkungen der Dienstleistungen sind zwei Beispiele der Qualitätsanforderungen der Freien Wohlfahrtspflege, wie sie in dem Arbeitspapier »Qualitätsziele der Wohlfahrtsverbände zur Erreichung ihrer spezifischen Dienstleistungsqualität « festgelegt sind. In dem Dokument vom Mai 2008 stellen die Wohlfahrtsverbände ihre Anforderungen an die Qualität ihrer angebotenen Dienstleistungen und Hilfen dar und operationalisieren diese für eine konkrete Überprüfbarkeit. Die Qualitätsziele - hier am Beispiel der Ziel- und Wirkungsorientierung - werden mit ihrer Operationalisierung für die Praxis umsetzbar, überprüfbar und nachweisbar, da die Prüfkriterien die gemeinsamen Anforderungen der Wohlfahrtsverbände an die Qualität ihrer Dienstleistungen und Hilfen konkretisieren und praxisorientiert formulieren. Das Arbeitspapier kann als PDF-Dokument kostenlos von der Website der Bundesarbeitsgemeinschaft der Freien Wohlfahrtspflege abgerufen werden.

Bundesarbeitsgemeinschaft der Freien Wohlfahrtspflege e. V., Oranienburger Straße 13-14, 10178 Berlin, Telefon 030 24089-0, Fax 030 24089134, E-Mail info@bag-wohlfahrt.de, Internet http://www.bagfw.de 
der Prozesse in Form einiger weniger Kennzahlen erfolgen. Kennzahlen verdichten die entsprechenden Informationen zu einer aussagefähigen Zahl und verdeutlichen damit einen größeren Zusammenhang. Damit der Erhebungsaufwand entsprechender Kennzahlen in einem angemessenen Verhältnis zu dem erwünschten Erkenntnisgewinn steht, müssen diese »smart« sein: »Smart« steht dabei für spezifisch, messbar, akzeptabel, realistisch und terminierbar. Es muss also konkret und eindeutig formuliert werden, was von wem gemessen werden soll, eine Messbarkeit als solche muss gegeben sein, die Messung muss bekannt und anerkannt werden (insbesondere von den Messenden), die Messung muss realisierbar sein, der Zeitpunkt der Messung und die Kommunikation der Messergebnisse muss terminierbar sein. Nur wenn diese Kriterien erfüllt sind, können entsprechende Kennzahlen zu den fünf pflegerischen Kernprozessen definiert und die Messungen regelmäßig durchgeführt und reflektiert werden.

Zur Vermeidung von Doppelstrukturen bietet es sich an, die Messung der pflegerischen Kernprozesse Sturzprävention, Dekubitusprävention, gewaltfreie Pflege, Prävention von Fehl- und Mangelernährung und professionelle Fallarbeit im Rahmen der Instrumente des Qualitätsmanagements durchzuführen und dann isoliert mit dem Management zu kommunizieren. Hierzu besonders geeignet ist die Pflegevisite, die ihrerseits bereits einen Bestandteil des Prozesses der professionellen Fallarbeit darstellt.

Pflegevisiten müssen in allen pflegerischen Einrichtungen durchgeführt werden, was bei MDK-Prüfungen auch kontrolliert wird. Unter einer Pflegevisite versteht man das Gespräch einer (in aller Regel leitenden) Pflegefachkraft mit dem Pflegekunden, das dem Ziel dient, die Pflegeprobleme und Ressourcen, die Pflegediagnostik, die Formulierung der Pflegeziele- und -maßnahmen sowie die Evaluation der Pflege zu überprüfen. Problemlos lassen sich bei Pflegevisiten Informationen auch zu den Themen Sturz, Dekubitus, gewaltfreie Pflege, Ernährung und Fallarbeit erheben und messen.

\section{Resümee und Warnung}

Die kennzahlenbasierte Auswertung zentraler pflegefachlicher Prozesse im Rahmen eines umfassenden Controllings erweitert das häufig einseitig finanzwirtschaftliche Controlling zu einem fachlichen Controlling und ermöglicht es der Unternehmensleitung, neben den Finanzen auch die Arbeitsinhalte im Blick zu behalten.

Diese Wahrnehmung stellt die Voraussetzung gezielter Interventionen dar, mit denen die Qualität weiterentwickelt und das Bestehen des Unternehmens am Markt gesichert werden.

Die Erkenntnisgewinnung durch das Controlling alleine kann dieses Ziel jedoch nicht erreichen: Zwar verändert eine Messung ihren Gegenstand selbst auch, doch stellt diese Veränderung keine strukturierte Weiterentwicklung dar, sondern schafft lediglich die Voraussetzung einer fachlichen Entwicklung, die von der Unternehmensführung initiiert werden muss.

\section{Was versteht man eigentlich unter einem Kern- prozess?}

Geschäftsprozesse eines Unternehmens werden in Kernprozesse, Management- und unterstützende Prozesse unterteilt. Der Kernprozess umfasst alle Tätigkeiten, die der direkten Erfüllung der Kundenbedürfnisse dienen. Er leitet sich aus der Kernkompetenz einer Organisation ab. Ein Kernprozess eines Automobil-Herstellers wäre beispielsweise »Autos produzieren«. Unterstützende Prozesse wären dabei beispielsweise »Buchhaltung « oder »Personalwesen $«$. Managementprozesse wären beispielsweise »Führung « oder »Qualitätsmanagement «. Der Begriff »Kernprozess « stammt aus dem Prozessmanagement und dem Qualitätsmanagement und wird auch in der Betriebswirtschaftslehre verwendet. Im Prozessmanagement steht der Kundenwunsch und dessen Erfüllung durch den Kernprozess im Mittelpunkt. Ein Unternehmen wird als Bündel von Kernprozessen verstanden, deren Sinn und Zweck die Erfüllung der Kundenanforderungen sind. Die Organisationsstruktur wird um 90 Grad gedreht, Aufbauorganisation und Ablauforganisation werden konsequent nach den Prozessen ausgerichtet. Kernprozesse beginnen mit dem Kundenwunsch - und enden mit der Erfüllung des Kundenwunsches. Sie integrieren alle dafür erforderlichen Teilprozesse. Schnittstellen zum Kunden und zu Lieferanten werden klar definiert.

Quelle: Wikipedia

(Internet http://de.wikipedia.org/wiki/Kernprozess)

\section{Korrektur}

Der Beitrag »Hohe Wertschöpfung « in SOZIALwirtschaft 3/2009 fasst die Ergebnisse eines Gutachtens über den ökonomischen und sozialen Wert Bürgerschaftlichen Engagements zusammen. Danach würden ohne Freiwillige die Kosten in vielen Einrichtungen explodieren, viele benachteiligte Menschen würden eine glaubwürdige Lobby verlieren und das soziale Klima würde kalt und rau. An zwei Stellen des Artikels haben sich leider bei der redaktionellen Bearbeitung Fehler eingeschlichen:

Erstens: Auf Seite 24 ist in der letzten Zeile von Abbildung 2 die Zahl 690.000 fälschlich von der vierten Spalte der vorherigen Zeile übernommen und in der zweiten Spalte wiederholt worden. Die Zahlen aus der dritten und vierten Spalte gehören in die zweite und dritte Spalte. Erst nach dieser Korrektur wird ein Ergebnis der Studie verständlich: »In der Stadt Würzburg stiftet der Einsatz von einem Euro für Engagement-Förderung einen Nutzen von 6,38 Euro.«

Zweitens: Auf Seite 25 müsste im vorletzten Absatz der letze Satz wie folgt lauten: »Die Beteiligten fordern wirkliche Teilhabe an politischen Entscheidungen, die für die Durchführung des Engagements relevant sind, nicht nur allgemeine Beteiligung.« 